\title{
Principios orientadores de la convivencia en el aula: una estrategia de reflexión colectiva'
}

Oscar Nail Kröyer"

Máximo Muñoz Reyes"

Jorge Gajardo Aguayo"

\section{Resumen}

El siguiente informe presenta los resultados de un estudio de caso sobre construcción colectiva de la convivencia en el aula, realizado en un establecimiento educacional público de la ciudad de Concepción, Chile. El objetivo general de la investigación es desarrollar a través de un proceso reflexivo y participativo, principios y valores orientadores de la convivencia en el aula, que creen las condiciones para el aprendizaje de los estudiantes. La importancia de la presente investigación radica en que la reflexión docente en materia de convivencia escolar es una necesidad en las unidades educativas, y su ausencia manifiesta múltiples tensiones: dialéctica entre cultura-contenidos, violencia simbólica, violencia escolar y dificultades para la gestión de aula. Frente a esto se propone una estrategia de reflexión colectiva, fundamentada en la recogida y análisis de datos mediante tres instrumentos, para su posterior discusión con los docentes de la comunidad educativa. Entre las principales conclusiones se encuentran que los sujetos participantes asignan una alta gravedad a los problemas que involucran al entramado humano en el aula, la necesidad de la construcción colectiva entre docentes de las normas para la convivencia en la sala de clases, y la importancia que tiene la gestión del equipo directivo en función de la mejora de la convivencia en el aula.

\section{Palabras clave}

Convivencia en el aula - Incidentes críticos - Reflexión docente.

I- Este artículo se desarrolla con financimiento del proyecto Anillos "Dialéctica de los aprendizajes y ruptura del círculo de bajos aprendizajes en sectores de alta vulnerabilidad", Proyecto CONICYT SOC-15.

II- Universidad de la Concepción, Concepción, Chile.

Contactos: onail@udec.cl; maximunoz@udec. cl; jorgegajardo@udec.cl 


\title{
Guiding principles of common life in the classroom: a collective reflection strategy'
}

Oscar Nail Kröyer"

Máximo Muñoz Reyes"

Jorge Gajardo Aguayo"

\begin{abstract}
This text reports on the results of a case study in the collective construction of common life in the classroom, conducted at a public education institute in the city of Concepcion, Chile. The general objective of the study is to develop through a reflexive and participative process guiding principles and values for the common life in the classroom, deemed to be conditions for students' learning. The importance of this investigation is rooted in the idea that teachers' reflection about common life at school is a felt need in education institutes, and that its absence gives rise to multiple tensions: dialectics between culture and contents, symbolic violence, school violence and difficulties of management in the classroom. In view of these facts we propose a collective reflection strategy founded on the collection and analysis of data through three devices, allowing their later discussion with teachers of the education community. Among the main conclusions of this study are: participants attributed high seriousness to the problems involving human relations in the classroom; the need for the collective construction between teachers of the norms for common life in the classroom; and the importance attributed to the management team for the improvement of common life in the classroom.
\end{abstract}

\section{Keywords}

Common life in the classroom - Critical incidents - Teacher reflection.

I- This article was developed with financial support from the Anillos project "Dialéctica de los aprendizajes y ruptura del círculo de bajos aprendizajes en sectores de alta vulnerabilidad", Project CONICYT SOC-15.

I- Universidad de la Concepción, Concepción, Chile.

contacts: onail@udec.cl; maximunoz@udec.cl; jorgegajardo@udec.cl 


\section{Introducción}

En Chile existe un currículo oficial, con códigos propios, que oblitera la relación dialéctica que se desarrolla en la sala de clases entre los contenidos y la situación cultural de cada estudiante que pertenece a bajos estratos sociales (CASTRO, 1981).

Lo anterior se ha convertido en un grave problema para la formación docente, puesto que la formación de profesores en Chile carece de un elemento educativo de carácter sociológico, primando más bien la instrucción de elementos relacionados con los contenidos y la formación de estos (ÁVALOS, 2004).

Esta ausencia en la formación del profesorado provoca una suerte de alienación del docente frente a sus estudiantes, puesto que en el aula, el primero observa en los segundos más bien carencias que diferencias. Esto se explica por la relación dialéctica entre cultura y contenido, que supone localizar al estudiante bajo una situación de déficit (BOURDIEU, 1977; BERNSTEIN, 1988). Tal situación altera la convivencia escolar del aula, ya que, al tratar de manera indistinta lo complementario de lo contradictorio al currículo, se desconoce la realidad situacional del educando. Lo anterior significa, por una parte, que no se incorpora al aula aquellos saberes propios del estudiante que podría complementar el currículo; pero también implica, por otra parte, no atender aquellos elementos contradictorios que no se adecúan al currículo, los que finalmente quedarán latentes.

Lo anterior se traduce en factor de inestabilidad para la convivencia en el aula, puesto que entre docente y estudiante se desarrolla una distancia latente, sobre la base de las relaciones dialécticas de tipo contradictorioantagónicas (CASTRO, 1981, 2007), explicadas en gran medida por las diferencias de códigos lingüísticos entre profesor y alumno. Estos códigos se despliegan como una dualidad de intercambios unidireccionales: mientras que el docente utiliza códigos lingüísticos elaborados -caracterizados por construcciones gramaticales complejas y el uso frecuente de pronombres impersonales, expresando significado independiente del contexto-, el estudiante de clase baja utiliza códigos lingüísticos restringidos -caracterizado por el uso de oraciones cortas y gramaticalmente simples, que expresan significados dependientes del contexto (AUSTIN, 2000; ÁVILA, 2005).

Por lo tanto, la escuela en su conjunto es un elemento que sanciona y legitima un sistema de hábitos y prácticas sociales, presentado como universales una serie de valores, desconociendo las particularidades de cada estudiante (ÁVILA, 2005).

\section{Situación de la convivencia y violencia escolar en Chile}

Lo anterior es sólo un enfoque explicativo que puede dar razón de la situación actual de la convivencia escolar en Chile. Si bien, esta es sólo una visión de las muchas que se podrían utilizar, consideramos que aporta suficientes aristas para analizar el tema, sirviendo de base para nuevos estudios teóricos y líneas de interpretación de los datos chilenos.

En los últimos años se han realizado en el país estudios significativos sobre violencia escolar. En este contexto, el año 2005 se efectúa el primer estudio nacional sobre convivencia escolar. El análisis de datos arrojó que, si bien el 91\% de estudiantes declaran sentirse bien en los establecimientos, un 28\% considera que ha recibido insultos y maledicencia, mientras que un $9 \%$ señala ser golpeado frecuentemente. A su vez, el 81\% de los docentes indican sentirse bien en el establecimiento, sin embargo, el $67 \%$ señala que la conducta más frecuente de maltrato desde los alumnos es faltarles el respeto (INSTITUTO IDEA, 2005). De igual modo, un estudio de carácter nacional dirigido por el Ministerio de Educación, revela que el 50\% de los estudiantes ha sido víctima de amenazas y hostigamiento de parte de sus pares, mientras que el $86 \%$ declara ser víctima de insultos y burlas (MINEDUC, 2010). 
Ahora bien, acotando el problema de la violencia escolar y vinculándolo con la convivencia desde un foco centrado en los docentes, el estudio VTR sobre bullying, ciberbullying y violencia escolar, desarrollado por Criteria Research (2010, p. 9) y apoyado por el Ministerio de Educación, indicó que “35\% de los estudiantes, reconoce que existen situaciones donde un grupo o un alumno han molestado a su profesor, ya sea porque 'se ríen de ellos', 'les rompen las cosas' o 'les faltan el respeto'”.

Del mismo modo, estudios cualitativos acerca de la autoridad pedagógica, entendida como un fenómeno social situado, indican que a diferencia de décadas anteriores, la autoridad no es algo que se constituye inmediatamente gracias a la escuela ni la universidad. Actualmente,

la autoridad se construye en la interacción social cotidiana con los alumnos y mediante un trabajo reflexivo y solitario sobre la práctica pedagógica por parte del profesor [...] en otros términos, el actor decisivo para construir la autoridad son los alumnos; ellos se convierten para los profesores en el principal y quizás único referente. (ZAMORA; ZERÓN, 2009, p. 179)

Por otra parte, estudios acerca de la mirada de los docentes sobre la violencia escolar, revelan, entre otros resultados, que los docentes

frente a sí mismos, [...] transparentan un cierto nivel de miedo frente a las familias y las autoridades. Se sienten solos y sin herramientas efectivas con qué combatir la situación, no percibiendo apoyo institucional. Confiesan desorientación e incapacidad para resolver el problema. (SAAVEDRA, VILLALTA; MUÑOZ, 2007, p. 57)

Con respecto al clima escolar, otros estudios realizados en Chile han demostrado que existe una relación entre intimidación y rendimiento académico, que puede o no estar mediada por éste (TOLED0; MAGENDZO; GUTIÉRREZ, 2009). Es así como en enseñanza primaria tal relación es directa, siendo poco significativa la mediación del clima. En cambio, en enseñanza secundaria, la relación entre intimidación y rendimiento académico es indirecta, siendo significativa la mediación del clima, ya que sólo a través de éste, la intimidación afecta el rendimiento. Sin embargo, dada la estructura vinculante percibida en enseñanza primaria entre intimidación y rendimiento, el clima deteriorado tendrá un efecto a largo plazo, que se manifestará en enseñanza media.

Frente a lo anterior, en la última década en Chile, se han concretado dos hitos en materia de políticas educacionales vinculadas con la temática de la convivencia escolar:

En el año 2002 el Ministerio de Educación publica la Politica de Convivencia Escolar, cuya finalidad es cumplir una "función orientadora y articuladora del conjunto de acciones que los actores emprenden y emprenderán a favor de la formación en valores de convivencia" (MINEDUC, 2002, p. 16-17). En una interesante propuesta, esta política plantea cuatro compromisos con el sistema educativo: 1) difundir y sensibilizar a distintos actores del sistema escolar sobre convivencia escolar en la formación ciudadana del estudiantado; 2) impulsar la readecuación del reglamento interno de convivencia con la participación de estudiantes, docentes, apoderados/as y codocentes; 3) capacitar en la resolución pacífica de los conflictos a actores claves del sistema, y por último, 4) Involucrar al Consejo Escolar con propuestas que mejoren el área curricular (MINEDUC, 2006).

Posteriormente en el año 2011, se aprueba la modificación de la Ley General de Educación (20.370) incorporando un párrafo sobre violencia escolar en su Título Preliminar. La finalidad de esta ley es establecer definiciones, responsabilidades, sanciones, y procedimientos que deben ser utilizados al enfrentar situaciones de violencia escolar, que afectan la convivencia escolar y de aula. 
Finalmente, el año 2012, el Ministerio de Educación, actualiza la Politica de Convivencia Escolar elaborada en el 2002, considerando los cambios sociales y culturales que ha experimentado Chile en materia de comunicaciones, tecnología, organización social e institución familiar (MINEDUC, 2012).

Entre otras acciones, la reformulación del año 2012 de la Política de Convivencia Escolar, propone dos aprendizajes interesantes, desde el punto de vista del trabajo reflexivo del profesorado: aprender a consensuar, vale decir a encontrar los aspectos comunes, que puede implicar pérdida y/o ganancia; $\mathrm{y}$ aprender a reflexionar, que implica repensar las acciones y sucesos, desde una perspectiva crítica, buscando la objetividad (MINEDUC, 2012).

Estas últimas perspectivas son interesantes, pues suponen situar al docente en el centro de la reflexión, llevándolo a ejercerla para repensar las situaciones desde una perspectiva más integradora.

\section{El docente y la gestión de la convivencia del aula: estilo,} reflexión y discurso

Los datos aportados por las investigaciones presentadas y las disposiciones adoptadas a nivel nacional, configuran el contexto sobre el cual el profesor chileno debe desenvolverse profesionalmente. Sin embargo, existen otros elementos, puestos en juego por el profesor, que configuran otro plano de acción. Con respecto a ellos, nos referiremos a tres que se encuentran directamente relacionados con la técnica de análisis de incidentes críticos y los objetivos de nuestra investigación: estilo, reflexión y discurso del docente.

Con respecto al estilo del docente y las dificultades asociadas, estudios indican que un proceder de enseñanza democrático tendría un efecto positivo en los rendimientos de los estudiantes. En cambio, un efecto contrario tendría un estilo autoritario. De la misma manera, un clima democrático estaría directamente correlacionado con el desarrollo de la autoestima de los estudiantes, mientras que un clima autoritario, está indirectamente relacionado a la violencia entre iguales. Esto implica que un profesor que no puede fomentar un clima de respeto en el aula, motivará en los estudiantes una mayor libertad para actuar contra sus pares (ESPINOZA, 2006).

Cuando se analizan los factores de intimidación y rol del docente, sepueden encontrar evidencias sobre la influencia que puede tener un profesor autoritario sobre los estudiantes, al actuar como un modelo de intimidación (YONEYAMA; NAITO, 2003). Igualmente, las conductas agresivas de los educandos pueden tener un origen en las dificultades de gestión de aula o en un desequilibrio hacia un exceso de lo normativo de parte de los docentes (OLWEUS, 1999). En este sentido, para Roland y Galloway (2002) la violencia de un grupo escolar se presenta con mayor probabilidad cuando estos son sancionados por los docentes de manera frecuente o controlados con normativas rígidas. Del mismo modo, mientras factores asociados a una gestión de aula y una estructura escolar deficientes contribuyen significativamente al acoso escolar, un abordaje claro de estos factores, y que implique a toda la escuela, tendrá efectos positivos y un impacto directo en la lucha contra la intimidación (ROLAND; GALLOWAY, 2002).

Considerando esto, se puede asumir que en gran medida, las acciones de todos los miembros de un aula afectarían los comportamientos de todos quienes están en ese ambiente (ALLEN, 2010). Sin embargo, serían los docentes quienes tienen que llevar a cabo la gestión de un clima de aula positivo, para promover una sana convivencia.

Es importante destacar que a pesar que la identidad docente se construye y reconstruye a lo largo del ejercicio profesional (ÁVALOS; SEVILLA, 2010), el aprendizaje de prácticas de gestión de aula se asocia incluso a antes del inicio de la carrera docente (ALLEN, 2010). De hecho, podemos encontrar por lo menos cuatro fuentes de origen de las técnicas de gestión 
de aula que utilizan los docentes en su labor profesional: la primera se asocia al aprendizaje temprano, basado en la observación de las prácticas de sus propios maestros en la edad escolar, las que perdurarán hasta los primeros años de ejercicio profesional (FAJET et al., 2005); la segunda se asocia a los centros de práctica, ya que la variedad y calidad de lo observado por los practicantes, tendrá un impacto en sus aprendizajes y acciones futuras. En este sentido el modelado que hagan los profesores guías será fundamental, pues, si es deficiente, ofrecerá un limitado conjunto de habilidades para la utilización ulterior (ALLEN, 2010); la tercera fuente se vincula a lo que los futuros profesores aprenderán en las aula universitarias; finalmente, una cuarta fuente, corresponde al aprendizaje que ocurre durante el ejercicio profesional de la docencia, destacando lo que Wenger, McDermott y Snyder (2002) denominan comunidades de práctica, donde los docentes comparten sus conocimientos, pero además, donde el aprendizaje se encuentra situado, lo que implica que tanto docentes como estudiantes, aprenden uno del otro.

Por otro lado, respecto al discurso y reflexión docente, las aportaciones de Paladino (2006) indican que los maestros deben habitualmente defender la validez de los principios y la normativa escolar. Para ello emplean argumentaciones éticas, discursos, "que como práctica social, implican una forma específica de interacción ante la presencia de una discrepancia o de un conflicto" (p. 21). Tal interacción se desarrolla en el aula a través de la combinación de razonamientos intuitivos y justificaciones argumentativas (TIRRI, 1995).

Al nivel del discurso docente en el aula, el tipo de relación comunicativa que el profesor establece con sus aprendices define su rol profesional, ya sea como guía de aprendizajes o conductor de contenidos, influyendo positiva o negativamente en la participación de sus estudiantes y su propia reflexión (CABRERA, 2003).
De este modo, si define un discurso bajo el rol de conductor, produce una comunicación de carácter lineal, de corte conductista, donde se representa al alumno como un individuo que debe comprender los mensajes, anulando la participación y la reflexión. En cambio, en el rol de guía de los aprendizajes, la comunicación o el diálogo es considerado como un proceso circular con significados compartidos que generan participación (CABRERA, 2003)

El profesor puede tomar estas dos opciones discursivas cuando interactúa en el aula con sus estudiantes al resolver situaciones conflictivas. Si decide dialogar con el conflicto, expresará una forma de equilibrio entre el cuidado, la justicia y la veracidad, a través de la buena voluntad, racionalidad y autonomía, buscando la participación real y la mejor solución, antes que el resultado inmediato. En cambio, si decide utiliza un discurso unilateral, priorizando la solución rápida con el fin de continuar la rutina sin la menor pérdida de tiempo, cerrará el diálogo propiamente tal, clausurando los contrastes (PALADINO, 2006).

La unidad ideal de diálogo sólo se manifiesta cuando se parte de un conflicto de acción identificado en común, así como de una cierta identidad de significados que permite dar sentidos parecidos a las mismas cosas. En los implicados se da una voluntad de intersubjetividad y de comprometerse en un proceso de diálogo; todos los participantes pueden argumentar y expresarse libremente de modo que no haya coacción y estén en pie de igualdad. La razón dialógica como elemento principal de dicha teoría ética supone que son justos aquellos principios y normas que se han conseguido por medio de un acuerdo basado en las mejores razones, expresadas entre todos los participantes o afectados en el conflicto. (PALADINO, 2006, p. 73-74) 


\section{Una estrategia de reflexión colectiva}

Frente a lo anterior, se infiere que en las escuelas de Chile existe una necesidad de reflexión docente colectiva, particularmente en materia de convivencia escolar.

Si consideramos que los factores de fondo que generan un deterioro de la convivencia escolar, se relacionan con cuestiones como las diferencias de códigos culturales y la relación dialéctica entre cultura y contenidos, entendemos que tales factores no se pueden abordar exclusivamente mediante disposiciones externas a los microsistemas. De la misma forma, se comprende que sólo a partir de la autonomía reflexiva de la organización, se pueden generar diferencias cualitativas en la apreciación de los problemas que afectan la convivencia en la escuela, puesto que la tendencia del profesor es mirar casi con exclusividad la cultura escolar oficial, como punto de partida de su accionar pedagógico.

Es por ello que la propuesta de trabajo utilizada aborda, en un primer momento, la recopilación de datos vinculados con los incidentes críticos más frecuentes que deben abordar los profesores, para, en un segundo momento, desarrollar una reflexión colectiva sobre tales incidentes, la forma de abordarlos y las posibles acciones a seguir.

Se entiende por Incidente crítico un hecho de la práctica profesional que nos causa perplejidad, produciendo sorpresa o inquietud por su falta de coherencia o por haber presentado resultados inesperados. Al mismo tiempo, obliga a tomar decisiones improvisadas, poniendo a prueba las habilidades de la persona.

La Técnica de Análisis de Incidentes críticos propiamente tal, tiene su origen en los Estados Unidos, en el marco de la Segunda Guerra Mundial, a partir de los estudios que Flanagan (1954, 1978) desarrollara para mejorar las habilidades de los pilotos de aviación. Para este autor la técnica sería una manera de recoger observaciones directas del comportamiento humano, a fin de facilitar su uso potencial en la solución de problemas prácticos. Posteriormente se extiende en diversos ámbitos y actividades. En el área educacional, existen muchas investigaciones y trabajos publicados sobre la práctica, aplicada a diversas situaciones y en diferentes contextos -sobre todo en formación inicial - (TRIPP, 1993; ESTRELA; ESTRELA, 1994; NAVARRO, LÓPEZ; BARROSO, 1998; ELÓRTEGUI, MEDINA; FERNÁNDEZ, 2003; MONEREO, 2010).

Esta técnica de análisis aplicada a la gestión de la convivencia del aula

identifica y reflexiona sobre las perturbaciones más comunes presentes en el aula, tales como conflictos, deterioro de las relaciones interpersonales, entre otras. Teniendo esto como finalidad mejorar la convivencia, particularmente entre docentes y alumnos, además de ayudar a una mejor gestión educativa del aula. (NAIL, 2010, p.12)

Siguiendo a Monereo (2010), la Técnica de Análisis de Incidentes críticos debería propiciar un cambio en la identidad profesional, vale decir, en las autorepresentaciones que tienen sobre sí mismos los docentes, que afectan tres aspectos fundamentales: a) el rol profesional, referido a las funciones que el docente considera debe desempeñar, con un fuerte componente institucional; b) la enseñanza y el aprendizaje, referido al conjunto de creencias $\mathrm{y}$ concepciones que poseen los docentes sobre qué significa enseñar y aprender una materia, especialmente la que ellos imparten; y c) los sentimientos asociados a la docencia, vinculados a los distintos procesos afectivos que impulsan y provocan la actuación docente, tanto de forma positiva (motivación), como negativa (inseguridad, vulnerabilidad) y que están vinculados al reconocimiento profesional, a la auto-evaluación personal y a la evaluación institucional. 


\section{Metodología}

Se diseñó una investigación para determinar los incidentes críticos más importantes en el área de convivencia escolar, en un total de cinco establecimientos de la provincia de Concepción, Chile.

Posteriormente se seleccionó un establecimiento, de los cinco, para trabajar en un ciclo reflexivo que incluyera la participación de estudiantes, familia y docentes, focalizándose en estos últimos. Participaron en total quince docentes, veinte familias y veinte estudiantes.

De esta manera, el presente estudio se clasifica como no experimental, ya que no existe selección aleatoria de los sujetos ni manipulación de variables, de tipo exploratoriodescriptivo, con un enfoque cualitativo. Se trabajó con variables de tipo nominal y ordinal.

El objetivo general de la investigación es desarrollar a través de un proceso reflexivo y participativo, principios y valores orientadores de la convivencia en el aula, que creen las condiciones para el aprendizaje de los estudiantes. Para su cumplimiento se desarrollaron los siguientes objetivos específicos: 1) Analizar los incidentes críticos de aula más representativos del establecimiento; 2) Identificar la gravedad que los actores le asignan a los comportamientos disruptivos de aula; 3) Construir en conjunto con los actores educativos, lo principios y valores orientadores de la convivencia de aula.

Dado el enfoque cualitativo de la investigación se construyeron los siguientes supuestos que orientaron la realización del estudio: 1) Los docentes no están preparados, teórica ni metodológicamente, en el manejo de incidentes críticos; 2) La reflexión colectiva acerca de la convivencia de aula tiene un papel fundamental en la prevención de problemas de convivencia; 3) El diálogo y la participación de los actores enfocado en la convivencia de aula ayuda a prevenir y construir la normativa escolar.

Para la recolección de la información se aplicaron tres instrumentos en fases consecutivas: el primer instrumento, entregado a los profesores, fue la Pauta de incidentes críticos de aula. Esta pauta debió ser contestada posteriormente a la ocurrencia de un incidente crítico al interior del aula. El instrumento consta de seis dimensiones: Descripción del caso; Emociones Despertadas; Actuación Profesional; Resultados de la actuación; Dilemas y Enseñanzas del caso y fue validado previamente por los investigadores.

Los docentes debían registrar los incidentes críticos ocurridos durante un periodo de tiempo de tres semanas.

Posteriormente se aplicó la Pauta de registro de disrupción de aula, cuyo fin era establecer la valoración que los actores educacionales dan a los comportamientos disruptivos en el aula. El instrumento consta de cuatro dimensiones: Conductas disruptivas relacionadas con las normas; Conductas disruptivas relacionadas con la tarea; Conductas disruptivas relacionadas con el docente; y Conductas disruptivas relacionadas con los compañeros. Este cuestionario de aplicó a docentes, estudiantes y familias.

Finalmente se aplicó el Cuestionario para la construcción democrática de normas, el cual está constituido de preguntas abiertas. Consta de 2 dimensiones: Identificación de las normas de centro y de aula y Construcción de normas de aula. Este cuestionario se aplicó los docentes, profesores y alumnos de la unidad educativa.

El análisis e interpretación de los datos se realizó según cada instrumento. Para la Pauta de Incidentes Críticos se realizó un análisis descriptivo, identificando los niveles en los cuales ocurren los fenómenos, los tipos de incidentes descritos, las emociones despertadas de forma más común, etc.

Por su parte, la Pauta de registro de disrupción de aula fue analizada de manera descriptiva de la pauta, calculando porcentajes de respuestas. Se construyeron gráficos de barras para facilitar la comprensión de los resultados.

Por último el cuestionario para la construcción democrática de normas fue analizado de manera descriptiva. Se identificaron 
las principales normas y elementos que para los docentes son significativos en la construcción de normas de aula.

Una vez analizada la información de los tres instrumentos, se procedió a establecer círculos de conversación con el profesorado para la mejora en la convivencia de aula y la construcción de normas para esta última. El análisis de esta etapa se focalizó en aquellos elementos que para los fines de la investigación eran fundamentales.

\section{Análisis descriptivo de las pautas de Incidentes críticos}

\section{a) Descripción de los casos}

Seis de los siete incidentes críticos que se presentan en este establecimiento están relacionados con alumnos que causan desorden al interior de la sala de clases, tal como: no trabajar en las labores asignadas por el profesor a los alumnos; pararse del puesto mientras el resto de los compañeros lee; jugar con celular durante el desarrollo de la clase; salir sin autorización de la sala de clases en repetidas ocasiones; no obedecer ante la solicitud de cambio de puesto dada por el profesor; e interrupción del inicio de la clase.

Se observa que los incidentes críticos que se presentan en este establecimiento educacional se relacionan fuertemente con disrupción de aula (ver tabla 1),

Tabla 1- Tipos de incidentes críticos de acuerdo a categorización

\begin{tabular}{c:c}
\hline Tipo de incidente crítico & $\begin{array}{c}\text { No }^{0} \text { de } \\
\text { casos }\end{array}$ \\
\hline Disrupción de aula junto con Violencia verbal & 0 \\
\hline Violencia física & 1 \\
\hline Violencia verbal & 0 \\
\hline Ambas (verbal más física) & 0 \\
\hline Disrupción de aula & 6 \\
\hline
\end{tabular}

En esta escuela se presenta un caso de violencia física, que ocurre en $5^{\circ}$ año básico, en el cual un alumno escupe a sus compañeros y al suelo. Se destaca esto porque este establecimiento es uno de los cuales se registraron episodios de violencia física entre alumnos. Además, llama la atención que el caso suceda entre los alumnos más pequeños de los cursos participantes de la investigación (ver Tabla 2).

Tabla 2 - Frecuencia de incidentes críticos de acuerdo al nivel

\begin{tabular}{c:c}
\hline Nivel & No de casos $^{\circ}$ \\
\hline 50 Básico & 1 \\
\hline 6 Básico & 2 \\
\hline 70 Básico & 0 \\
\hline 8 Básico & 4 \\
\hline TOTAL & 7 \\
\hline
\end{tabular}

La descripción de los casos menciona que en ninguno de estos las medidas tomadas por los docentes fueron efectivas, ya que los alumnos continuaron con su comportamiento, no trabajando o no acatando las órdenes de sentarse y trabajar o de limpiar el piso en al caso del alumno que escupió.

Las acciones tomadas ante los incidentes críticos son mencionadas solo por un profesor, en el caso del alumno que juega con el celular, el cual fue expulsado de la sala.

\section{b) Emociones despertadas}

Las emociones que despiertan los incidentes críticos tienen dos sentidos: los docentes describen las emociones que en ellos despertó el incidente crítico (ver Tabla 3) y las que ellos observan en el alumno involucrado (ver Tabla 4). 
Tabla 3- Frecuencia de emociones que los docentes dicen sentir

\begin{tabular}{c|c}
\hline Emoción despertada & No $^{\mathbf{0}}$ de veces mencionada \\
\hline Rabia & 1 \\
\hline Impotencia & 3 \\
\hline Indignación & 1 \\
\hline
\end{tabular}

Tabla 4- Frecuencia de emociones que los docentes observan en sus alumnos

\begin{tabular}{|c|c|}
\hline Emoción despertada & $\mathrm{N}^{0}$ de veces mencionada \\
\hline Rebeldía & 3 \\
\hline Desprecio & 2 \\
\hline
\end{tabular}

Los sentimientos de rabia e impotencia, que son mencionados con mayor frecuencia, están vinculados al accionar de los alumnos que no trabajan en la sala de clases, puesto que causan desorden haciendo caso omiso de las indicaciones del profesor. Se relaciona con estas emociones cierta pérdida de autoridad por parte de los docentes al interior de las aulas, ya que no logran que los alumnos causantes de los incidentes críticos cesen en su comportamiento y retomen las labores asignadas. Además sienten impotencia al no poder resolver la situación conflictiva y cómo esto repercutirá en el proceso de aprendizaje del alumno.

Las emociones que los docentes atribuyen a los alumnos son rebeldía, al no querer aceptar lo que se les ordena cumplir y al ser sancionados por su acción. Un profesor manifiesta la actitud de rechazo del curso ante el comportamiento del alumno, en el caso de la violencia física: "La actuación del alumno fue despectiva y rebelde mostrándose como un líder negativo del curso. El curso mostró rechazo a su actitud" (Pauta de Registros de incidentes críticos no 2. Profesor, 1). Este profesor además menciona que el alumno se desenvuelve como un líder negativo del curso, lo cual implica que tiene algún tipo de influencia sobre sus compañeros.

\section{c) Actuación profesional}

Las actuaciones de los docentes se centran en un primer momento en contener el mal comportamiento del alumno mediante el diálogo, y la advertencia de una posible sanción. Las sanciones aplicadas son: anotación en la hoja de vida del alumno, expulsión de la sala pidiendo ayuda al paradocente, citación del apoderado, ordenar que el estudiante realice el trabajo en el pasillo con su silla y mesa (acuerdo de los profesores). Además un docente manifiesta que le "hace ver" al alumno la poca valorización que tiene del trabajo del resto de sus compañeros y del trabajo del profesor.

Un docente señala no haber aplicado sanción, sólo conversó con los alumnos involucrados.

Como resultado de las actuaciones mencionadas, cuatro docentes indican que el curso se mantiene expectante frente al desarrollo de la relación docente-alumno. En dos de estos casos los docentes indican que el resto del grupo rechazó el comportamiento del estudiante. En los otros dos casos sólo se menciona la atención del curso, no especificando en que concluyó ésta.

El docente que expulsó al alumno de la sala por utilizar el celular indica que al cumplirse esta sanción el curso se tranquilizó. En el incidente del alumno que no quiso cambiarse de puesto según lo ordenó el profesor, a este se le anotó en el libro lo que tuvo como efecto que el alumno dejara de molestar.

Finalmente un docente indica que a pesar de la sanción (alumno expulsado de la sala) este no trabajó en la labor asignada, dedicándose a otras cosas.

Se desprende de este análisis que la actuación del docente, no involucra sólo al alumno relacionado con el incidente crítico, sino que además provoca un efecto en el curso, el cual espera ver de qué manera se resolverá la situación. La aplicación de sanciones tuvo efectos dispares, en cuanto que en algunos casos cumplió su cometido de resolver la situación y 
en otros su aplicación no tuvo mayor impacto en el comportamiento del alumno.

\section{d) Dilemas}

La mayoría de los docentes indica preguntas respecto a su actuación puntual en los incidentes críticos, tales como: ¿Valió la pena sacarlo de la sala?; ¿Cambiará su comportamiento cuando este tipo de conducta viene repitiéndose desde el año anterior?; ¿Qué habría pasado si el alumno contesta cuando los alumnos restantes le decían que su proceder no era el correcto?; ¿Qué habría pasado si no le llamo la atención cuando salió?; ¿El resto del curso se sentiría con las mismas prerrogativas para realizar la misma acción? Los docentes se preguntan sobre las implicancias de sus decisiones y el efecto que estas tendrán en el alumno y en el curso. Además se cuestionan si es que otra decisión hubiese sido la indicada.

\section{e) Enseñanzas del caso}

Los docentes indican que los incidentes críticos les permitieron aprender que deben utilizar estrategias para que los alumnos mejoren su conducta, como la asignación de roles que les permitan participar más en clases. Además los incidentes críticos pueden ayudar a hacer comprender a los alumnos que las normas y el reglamento de convivencia deben ser respetados, y cuando no sea así, se recibirá sanción.

Un docente indica que aprendió que se hace necesario separar antes del inicio de la clase a los alumnos que se sabe puedan causar problemas, para así evitar sucesos que impacten en el proceso de enseñanza aprendizaje.

\section{Gravedad de las acciones de disrupción}

Posteriormente, se procedió a consultar a las familias, docentes y estudiantes sobre la gravedad que le asignan a una serie de comportamientos disruptivos y violentos en el aula de clases. Esto se realizó con el fin de identificar cuales son los comportamientos de los estudiantes en la sala de clases a los cuales una parte significativa de la comunidad educativa asigna mayor gravedad. Se observa que para los sujetos, las acciones de mayor gravedad están vinculadas a la relación estudiante-profesor y estudiante-estudiante. A continuación se presentan los gráficos, por cada dimensión y pregunta del instrumento. Para fines del estudio, se presentarán solo los porcentajes totales de respuesta (docentes, estudiantes y familias) asociados a la opción de respuesta Alta gravedad.

Gráfico 1- Frecuencia de respuesta de para preguntas relacionadas a las normas

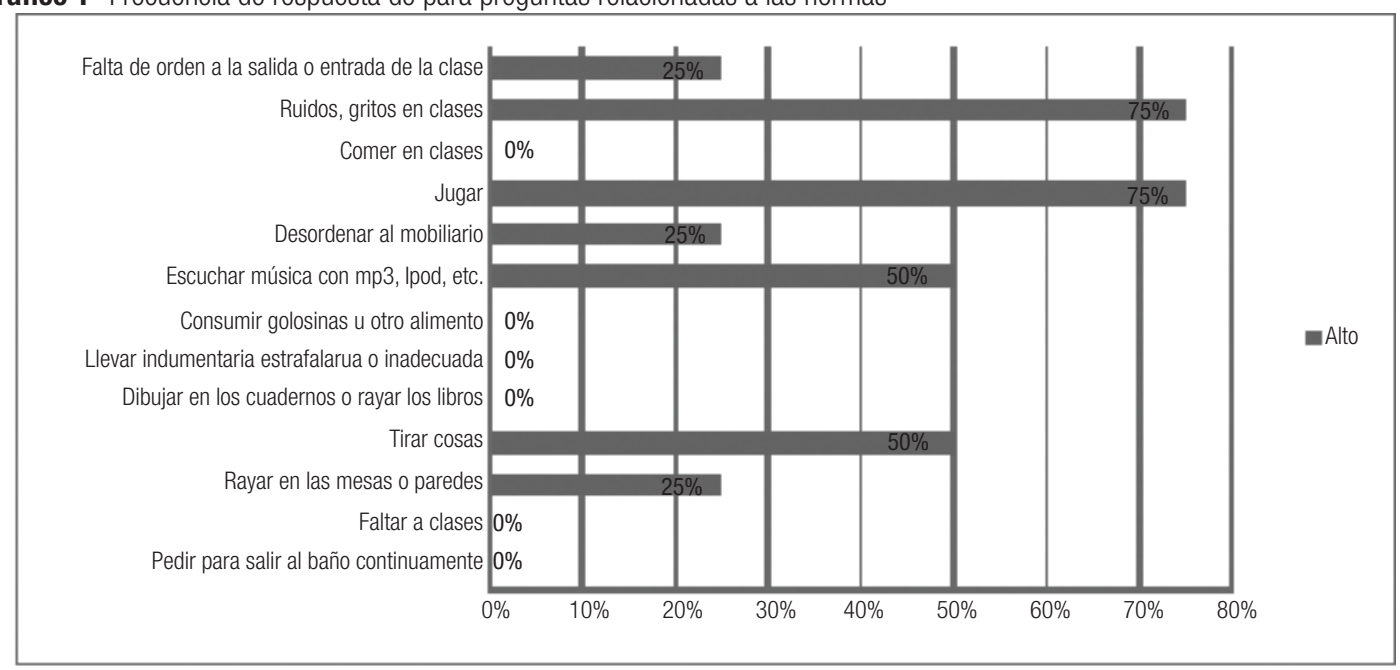


Se observa en el gráfico 1 que las acciones más graves son: ruidos o gritos en clases y jugar en clases. Se destaca también la baja importancia que los sujetos le dan a muchas de las acciones tipificadas en esta dimensión.
Al consultarles sobre la gravedad de las acciones respecto de la tarea (gráfico 2), se observa que el rehusar hacer la tarea en clase es la que tiene un mayor porcentaje de sujetos que la mencionan de alta gravedad.

Gráfico 2- Frecuencia de respuesta para preguntas relacionadas a la tarea

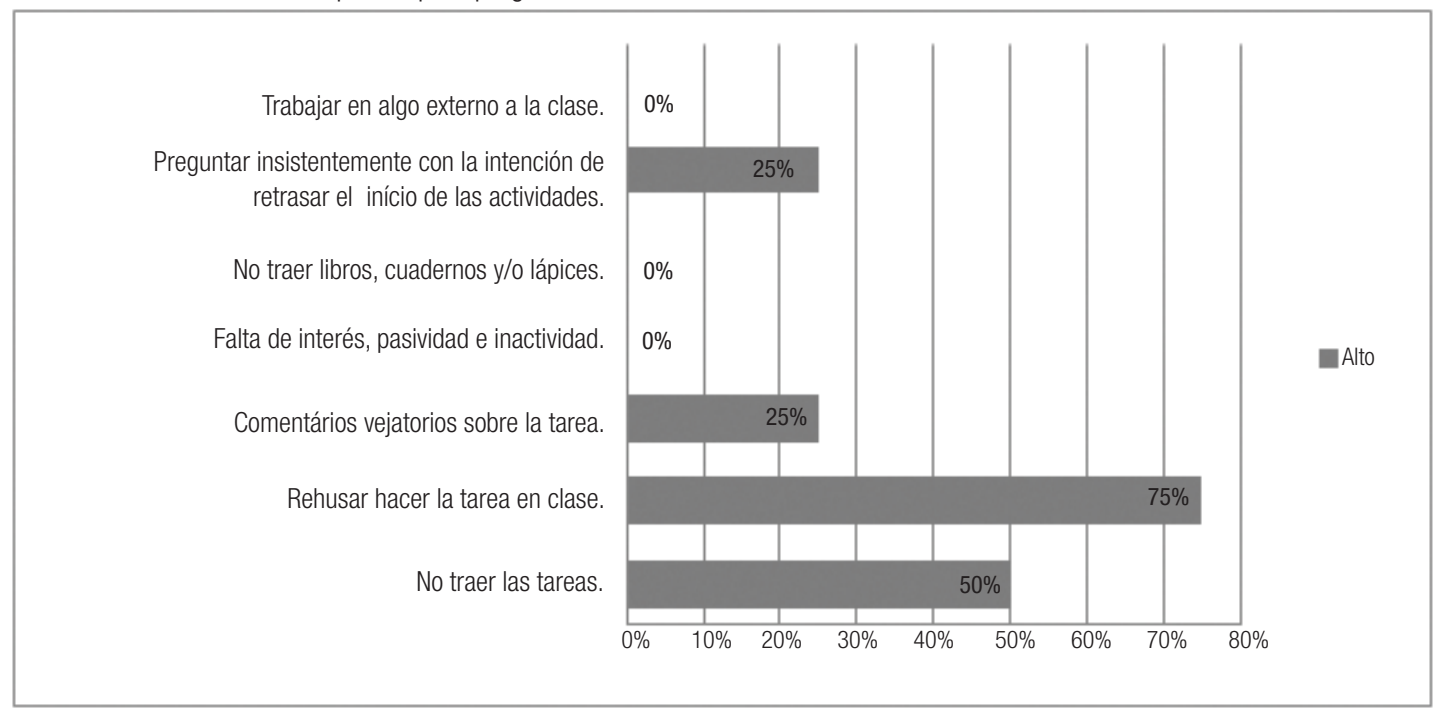

$\mathrm{Al}$ ser consultados sobre la gravedad de las acciones disruptivas vinculadas a la relación docente-estudiante (gráfico 3), se observa que tres de estas son para todos los sujetos, de alta gravedad: golpear el profesor, amenazar al profesor y no acatar sus órdenes. Nótese que dos de estas acciones, involucran algún grado de violencia.

Gráfico 3- Frecuencia de respuesta para preguntas relacionadas con el docente

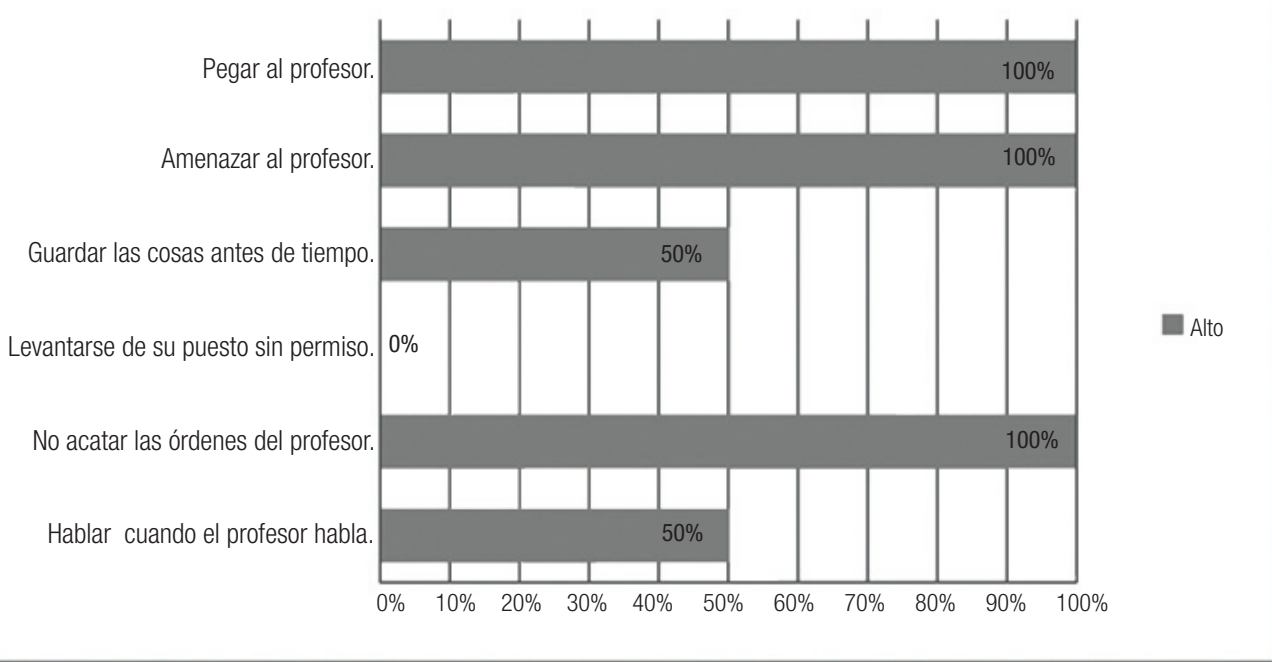


Gráfico 4- Frecuencia de respuesta para preguntas relacionadas con la relación con el compañero

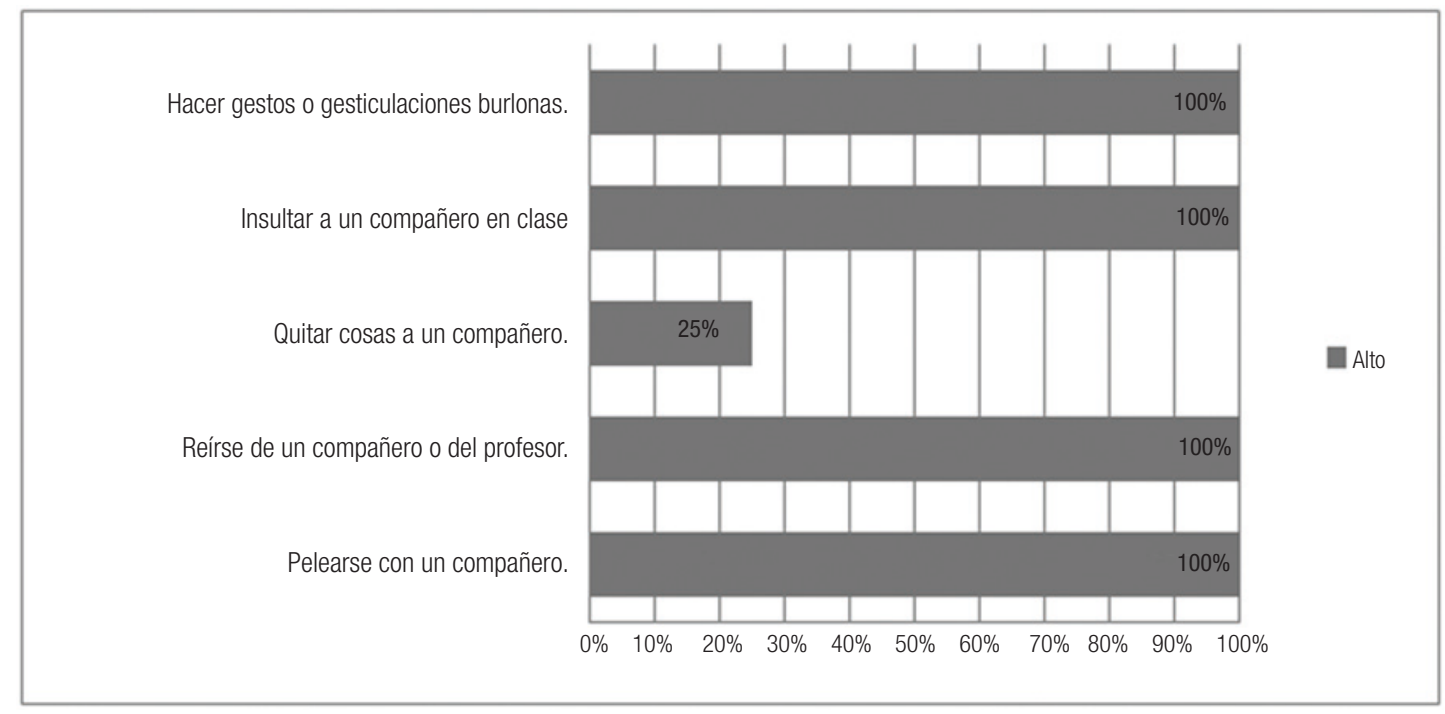

Finalmente, respecto a la relación entre estudiantes (gráfico 4) se observan cuatro ítems que son mencionados por todos los sujetos como de alta gravedad: hacer gesticulaciones burlonas; insultar a un compañero en clase; reírse de un compañero o del profesor; pelearse con un compañero. Nuevamente, y al igual que en la dimensión anterior, las acciones que todos los sujetos consideran de gravedad implican algún grado de violencia.

\section{Cuestionario para la construcción democrática de normas de aula}

$\mathrm{Al}$ ser consultados sobre el conocimiento y fundamento de las normas de convivencia de centro y de aula, las respuestas son divergentes. Se debe destacar que se hace una diferencia en las preguntas entre: Normas de Centro, que deben ser cumplidas en todas las locaciones del establecimiento, y Normas de Aula, aquellas que deben ser cumplidas con particular exigencia en la sala de clases, y que se vinculan directamente el proceso de enseñanza-aprendizaje que se realiza en el establecimiento.

Por un lado los alumnos se focalizan en las normas que están orientadas a la presentación personal, tales como: usar delantal, usar uniforme, no andar con zapatillas. Por otro lado, los estudiantes al referirse a las normas que posibilitan/limitan las relaciones interpersonales lo hacen de manera genérica: no pelear con compañeros, no decir garabatos, no gritar, sin profundizar en cuáles son los fundamentos de existencia y de que manera han sido construidas.

Se infiere desconocimiento por parte de los estudiantes de la razón de ser de las normas al interior de los establecimientos y a la vez, se observa que las reglas que ellos identifican están formuladas en sentido negativo, negando o prohibiendo comportamientos, sin identificar ningún tipo de norma que incentive o premie los comportamientos esperados y/o deseables en los miembros de la comunidad educativa.

Por su parte, las familias al igual que los estudiantes, no son capaces de identificar con precisión las normas del centro. Tampoco son capaces de expresar los fundamentos de las normas existentes en el establecimiento. Mencionan normas como: Respeto del uno al otro,Respeto entre sí, Comportarse en el comedor, Llegar a la hora. Si bien este tipo de conductas son deseables, las familias no saben si son normas de centro. Además, tampoco son capaces de diferenciar cuáles se 
refieren al comportamiento de los estudiantes al interior de la sala de clases y cuáles son aplicables a la totalidad del centro. Sin embargo, y a diferencia de lo anterior, las familias tienden a redactar las normas que dicen conocer de manera positiva, indicando cual es el comportamiento deseado (hacer), en vez de formularlas en negativo (no hacer).

Respecto del fundamento de la existencia de normas en el centro escolar, las familias mencionan que sirven para controlar la convivencia, para entregar valores a los estudiantes, para desarrollar la tolerancia, respeto mutuo, entre otras. Se infiriere que las familias de los estudiantes dan sentido a las normas del centro en cuanto ayudan a regular la convivencia escolar entre los actores y a la vez, preparan para la vida en sociedad. Sin embargo, ninguna familia mencionó que el principal aporte de las normas de convivencia es que permiten regular los comportamientos para facilitar los aprendizajes de los niños, función primaria de todo centro educativo.

Finalmente los docentes, al igual que el resto de los actores del centro educativo, identifican genéricamente normas, pero no son capaces de distinguir entre normas de centro y normas de aula de clases. Particular atención merece que para los docentes muchas normas que pueden ser consideradas de centro, se les agrega en la sala de clases y quedan definidas como normas de aula; ejemplo de esto es lo siguiente: no utilizar celular, la cual es mencionada por un docente como norma de centro. Otro docente, sin embargo, menciona: no utilizar celular en la sala de clases, observándose que para algunos docentes, no existe diferencia significativa entre las normas que son exigibles a toda la comunidad educativa y aquellas que son exigibles en la sala de clases y que median entre la relación docente-estudiante, facilitando o entorpeciendo el proceso de enseñanza-aprendizaje.

Respecto de la utilidad de la norma, los docentes tienden a identificar de mejor manera sus funciones y los fundamentos. Expresan que son necesarias porque regulan y facilitan las relaciones al interior del establecimiento entre todos los actores de la comunidad educativa. Además, vinculan las normas del centro educativo a las normas existentes en la sociedad, señalando que facilita la adecuada integración y desenvolvimiento de los estudiantes en la comunidad extra-escolar. Sin embargo, los docentes no explicitan que las normas de convivencia tanto de centro como de aula tienen como función primordial facilitar el proceso de aprendizaje de los estudiantes. $\mathrm{Al}$ ser consultados sobre esto, señalan que ellos saben que es así y consideran que esto es entendido por la totalidad de la comunidad educativa, sin embargo y analizando las respuestas dados por los otros actores (alumnos y familias) este sentido, que para los docentes es implícito, no llega a ser conocido o comprendido.

\section{Círculos de conversación}

Una vez analizada toda la información recogida, se procedió a dar a conocer los resultados a los docentes, para dialogar y discutir ciertas cuestiones que para fines de la investigación resultaban esenciales.

Por un lado se conversó la necesidad de explicitar el sentido y la función de las normas. Se comprendió que toda la comunidad educativa debe estar en conocimiento que el fin primordial de las normas de convivencia es facilitar el proceso de enseñanza-aprendizaje, sin desmerecer el resto de las funciones que los actores de la comunidad educativa también les atribuyen. Este principio fue compartido por todos los docentes participantes.

Otro elemento significativo que se discutió, y que surgió de los propios docentes, es la necesidad de trabajar coordinados con respecto a las normas de aula. Muchos docentes señalan que ellos tienen normas para la sala de clases y que las dan a conocer al alumnado, sin embargo estas reglas no son conocidas por el resto de los docentes y algunas veces entran en contradicción, causando confusión entre 
el estudiantado. Por lo tanto, se reconoció la necesidad de establecer una base fundamental de normas para la sala de clases, que sea exigible en todas las asignaturas, sin desmerecer que dependiendo de la particularidad de la clase que se realiza se pueden generar algunas específicas por parte de cada docente.

Finalmente, se dialogó sobre la necesidad de dar a conocer a la comunidad educativa las normas de convivencia y la importancia de que estas sean cumplidas. En este punto, algunos docentes sugirieron modificarlas o agregar nuevas normas en el sentido contrario al actual, es decir potenciando los comportamientos positivos antes que prohibir los negativos. En este punto se generó consenso entre los docentes respecto de la necesidad de construir un reglamento de convivencia escolar, que por un lado especifique las normas de aula y por otro, traslade su enfoque desde lo punitivo a lo formativo. Para los docentes la idea es potenciar el hacer, antes que enfatizar el no-hacer.

\section{Conclusiones}

Existe un alto nivel de coincidencia entre los actores al considerar de alta gravedad los problemas de aula que implican las relaciones alumno-alumno y alumno-profesor, replicando la información que las investigaciones a nivel nacional han destacado en cuanto a convivencia y violencia escolar (INSTITUTO IDEA, 2005; MINEDUC, 2010; CRITERIA RESEARCH, 2010). Sin embargo un elemento importante que adquiere relevancia en la calidad de tales relaciones es la normativa de la escuela.

$\mathrm{Si}$ consideramos que el reglamento de convivencia de los establecimientos educacionales es una herramienta fundamental para la observación de la convivencia escolar, se debe tener presente que una de sus funciones es mejorar el entramado humano al interior de las aulas, normando y posibilitando las relaciones interpersonales entre los sujetos.

Por ello, para tener una reflexión significativa de las normas, es necesario primariamente levantar un diagnóstico con datos empíricos, que permita visibilizar los problemas más importantes y urgentes de la convivencia escolar en los establecimientos. Este aporte de datos empíricos permitiría impulsar adecuadamente la validación de un reglamento participativo de convivencia, tal como lo han sostenido reiteradamente las políticas de convivencia escolar desarrolladas en el país (MINEDUC, 2002, 2012). Al respecto, en este estudio de caso, la recogida y análisis de datos movilizó un mejor proceso reflexivo por parte de los docentes de la comunidad educativa y facilitó el diálogo y la discusión. Por lo tanto, se requiere que los establecimientos cuenten con las herramientas y el recurso humano capacitado necesario para realizar este proceso de diagnóstico de la convivencia escolar.

Además, resulta significativo el lograr que al interior del establecimiento los docentes construyan normas de aula sobre la base del consenso. No se trata en este caso de negar las particularidades en la normativa que cada docente aplica, sino que más bien de construir un piso común de normas de aula sobre el cual los docentes que lo deseen puedan agregar normas particulares. Igualmente necesario es que la redacción de éstas sea en términos positivos, fomentando y premiando el buen comportamiento, antes que en términos punitivos que busquen el castigo del infractor.

Nuevamente los espacios de reflexión y diálogo son cruciales para alcanzar estas metas, puesto que sólo a partir de la reflexión, es posible dialogar con los conflictos en el aula, de tal modo de lograr un equilibrio entre la norma, el estudiante y el propio docente. Por ello, la gestión de las normas a nivel institucional requiere de un proceso de coordinación desde la dirección de los establecimientos, vinculando a docentes, estudiantes y familias. El éxito del análisis y la reflexión de la normativa escolar está asociada a la gestión institucional que se realiza en función de esto. En consecuencia, es necesario que el establecimiento organice la estructura y los espacios de diálogo para poder analizar el 
funcionamiento de la normativa escolar y el estado de la convivencia. Esto implica un desafío a la institución respecto de su organización y la importancia que debe dar a este trabajo.

Del mismo modo, se considera significativo desarrollar medidas fundamentadas en el diálogo entre los actores del establecimiento, ya que según este estudio existe una coincidencia en que los problemas más graves que pueden presentarse en el aula son los referidos a la dimensión relaciones humanas, por lo tanto la mejora no pasaría por acciones punitivas o por agregar o disminuir las normas en el reglamento, sino que por un mejoramiento cualitativo de las relaciones profesor-alumno y/o alumno-alumno, donde adquiere relevancia el diálogo, la participación democrática, liderazgo compartido y la toma de decisiones basadas en datos empíricos.

Este diálogo entre los actores es fundamental para construir un equilibrio entre el cuidado, la justicia y la veracidad (PALADINO, 2006), evitando así las soluciones emergentes e inmediatistas que clausuran los contrastes. Con ello se abren posibilidades de superar aquella distancia latente que se ha gestado entre estudiantes y docentes, que evita incorporar lo complementario al currículum, atendiendo los aspectos contradictorios. 


\section{Referencias}

ALLEN, Kathleen. Classroom management, bullying, and teacher practices. The Professional Educator, Rochester, v. 34, no 1, p. 1-15, 2010. Disponible en: <http://www.theprofessionaleducator.org/articles/Allen_final.pdf>. Acceso en: 5 jun. 2012.

AUSTIN, Tomás. Fundamentos sociales y culturales de la educación. Chile: Editorial Universitaria Arturo Prat, 2000.

ÁVALOS, Beatrice. La formación docente inicial en Chile. Digital observatory for higher education in Latin America and the Caribbean. UNESCO, IESALC. Santiago de Chile, 2004. Disponible en: <http://www.iesalc.unesco.org.ve >. Acceso en: 5 jun. 2012.

ÁVALOS, Beatrice; SEVILLA, Alejandro. La construcción de la identidad profesional en los primeros años de docencia: evidencia desde la investigación. Centro de investigación avanzada en educación, Santiago de Chile, p. 1-27, 2010. Disponible en: <http://nucleodocentes.cl/img_page/bajando.php?archivo=Avalos-Sevilla.pdf>. Acceso en: 5 jun. 2012.

ÁVILA, Mercedes. Socialización, educación y reproducción cultural: Bourdieu y Bernstein. Revista Interuniversitaria de Formación del Profesorado, Zaragoza, v. 19, n. 1, p. 159-174, 2005. Disponible en: <http://redalyc.uaemex.mx/ redalyc/pdf/274/27419109.pdf>. Acceso en: 5 jun. 2012.

BERNSTEIN, Basil. Poder, educación y conciencia. Sociología de la transmisión cultural. Santiago de Chile: CIDE, 1988.

BOURDIEU, Pierre. La reproducción. Barcelona: Editorial Laia, 1977.

CASTRO, Abelardo. Det institutionaliserade övervaldet. Estocolmo: Pedagogiska Institutionen Stockholms Universitet, 1981, 240. Tesis de Licenciatura en Educación (C-uppsats).

Formación inicial y profesión docente. In: BRUNNER, J.; PEÑA, C. La reforma al sistema escolar: aportes para el debate. Santiago de Chile: Universidad Diego Portales, 2007, p. 103-120.

CABRERA, Jessica. Discurso Docente en el Aula. Estudios Pedagógicos, Valdivia, n. 29, p. 7-26, 2003. Disponible en: <http://www.scielo.cl/scielo.php?pid=S0718-07052003000100001\&script=sci_arttext>. Acceso en: 11nov. 2012.

CRITERIA Research. Estudio VTR sobre bullying, ciberbullying y violencia escolar, Santiago de Chile, p. 112, 2010. Disponible en: <http://criteria.cl/wp-content/themes/criteria/archivos/ESTUDI0-Bullying-y-Ciberbullying.pdf>. Acceso en: 5 jun. 2012.

ELÓRTEGUI, Nicolás; MEDINA, Mercedes; FERNÁNDEZ, José. Los "incidentes críticos" en la formación y perfeccionamiento del profesorado de secundaria de ciencias de la naturaleza. Revista Interuniversitaria de Formación del Profesorado, Tenerife, v. 46, p.101-112, 2003.

ESPINOZA, Evelyn. Impacto del maltrato escolar en el rendimiento académico. Revista Electrónica de Investigación Psicoeducativa, Guatemala, v. 4, n. 9, p. 221-238, 2006. Disponible en: <http://www.investigacion-psicopedagogica. org/revista/new/ContadorArticulo.php?64>. Acceso en: 5 jun. 2012.

ESTRELA, María Teresa; ESTRELA, Albano. Técnica dos incidentes críticos no ensino. Lisboa: Estampa, 1994.

FAJET, Walter. et al. Preservice teachers'perceptions in beginning education classes. Teaching and Teacher Education, Miami, v. 21, p. 717-727, 2005.

FLANAGAN, John. The critical incident technique. Psychological Bulletin, Pittsburgh, v. 51, n. 4, p. 327-358, 1954. 
FLANAGAN, John. A research approach to improving our quality of life. American Psychologist, Washington, DC, v. 33, p. 138-147, 1978.

INSTITUTO IDEA. Estudio de convivencia escolar. La opinión de estudiantes y docentes. Informe final. Santiago de Chile: Ministerio de Educación, UNESCO, 2005.

MINEDUC - Ministerio de Educación de Chile. Política de convivencia escolar. Santiago de Chile, 2002. Disponible en: http://www.mineduc.cl/biblio/documento/Politica_Convivencia.pdf Acceso en: 05-06-12.

Conceptos clave para la resolución pacífica de conflictos, en el ámbito escolar. Santiago de Chile, 2006. Disponible en: <http://www.educarchile.cl/UserFiles/P0001/File/CR_Articulos/resolucion\%20pacifica\%20de\%20 conflictos.pdf>. Acceso en: 5 jun. 2012.

Bullying, un enemigo oculto en nuestras escuelas. Santiago de Chile, 2010. Disponible en: <http://www. mineduc.cl/usuarios/mineduc/doc/201104251732440.ENCUESTA\%20BULLYING.pdf>. Acceso en: 5 jun. 2012.

Política nacional de convivencia escolar. Santiago de Chile, 2012. Disponible en: <http://www.mineduc. cl/usuarios/convivencia_escolar/doc/201203262303500.PoliticadeConvivenciaEscolar.pdf>. Acceso en: 5 jun. 2012.

MONEREO, Carles. La formación del profesorado: una pauta para el análisis e intervención a través de incidentes críticos. Revista Iberoamericana de Educación, Madrid, v. 52, p. 149-178, 2010.

NAIL, Oscar. Los incidentes críticos de aula. Un aporte a la gestión docente y la formación inicial. Proyecto DIUC (Dirección de investigación Universidad de Concepción) 2010-2011 (código 210.161.006-1.0.). Concepción: Universidad de Concepción, 2010.

NAVARRO, Rosario; LÓPEZ, Antonia; BARROSO, Purificación. El análisis de incidentes críticos en la formación inicial de maestros. Revista Electrónica Interuniversitaria de Formación del Profesorado, Zaragoza, v. 1, n. 1, p. 1-14, 1998. Disponible en: <http://aufop.com/aufop/uploaded_files/articulos/1224541140.pdf>. Acceso en: 5 jun. 2012.

OLWEUS, Dan. Sweden. In: SMITH et al. (Orgs.). The nature of school bullying: A cross-national perspective. London \& New York: Routledge, 1999, p. 7-27.

PALADINO, Celia. Conflictos en el Aula. Perspectivas y voces docentes. Argentina: Miño y Dávila editores, 2006.

ROLAND, Erling; GALLOWAY, David. Classroom influences on bullying. Educational Research, UK, v. 44, n. 3, p. 299-312, 2002.

SAAVEDRA, Eugenia; VILLALTA, Marco; MUÑOZ, María Teresa. La violencia escolar: la mirada de los docentes. Límite, Tarapacá, v. 2, n.15, p. 39-60, 2007.

TIRRI, Kirsi (Org.). Effectiveness of teacher education. New challenges and approaches to evaluation. Reports from the Department of Teacher Education in Tampere University, Finlandia, 1995.

TOLED0, María; MAGENDZO, Abraham; GUTIÉRREZ, Virna. Relación entre intimidación (bullying) y clima en la sala de clases y su influencia sobre el rendimiento de los estudiantes. Proyecto FONIDE n. 32, Ministerio de Educación, Santiago de Chile, 2009.

TRIPP, David. Critical incidents in teaching: developing profesional judgment. Nueva York: Routledge, 1993.

WENGER, Etienne; MCDERMOTT, Richard; SNYDER, William. A guide to managing knowledge: Cultivating communities of practice. Boston: Harvard Business School, 2002.

YONEYAMA, Shoko; NAITO, Asao. Problems with the paradigm: the school as a factor in understanding bullying (whit spacial reference to Japan). British journal of Sociology of Education, UK, v. 24, n. 3, p. 315-330, jul. 2003. 
ZAMORA, Guillermo; ZERÓN, Ana. Sentido de la autoridad pedagógica actual. Una mirada desde las experiencias docentes. Estudios Pedagógicos, Valdivia, v. 35, n. 1, p. 171-180, 2009.

Recebido em:06.06.2012

Aprovado em: 10.12.2012

Oscar Nail Kröyer es Doctor en Educación. Psicólogo y profesor de Filosofía de la Universidad de Concepción, Chile. Profesor Asociado, Director del Departamento de Ciencias de la Educación, Facultad de Educación, Universidad de Concepción, Chile.

Máximo Muñoz Reyes es Magíster en Política y Gestión Educacional. Licenciado en Sociología de la Universidad de Concepción, Chile. Facultad de Educación, Universidad de Concepción, Chile.

Jorge Gajardo Aguayo es Magíster en Gestión, Liderazgo y Política Educativa. Licenciado en Educación, profesor de Filosofía de la Universidad de Concepción, Chile. Asesor en Unidad de Investigación y Desarrollo Docente, Dirección de Docencia, Universidad de Concepción, Chile. 
